\title{
Navigation With a Passive Brain Based Interface
}

\author{
Jan B.F. van Erp \\ TNO Human Factors \\ Kampweg 5 \\ 3769ZG Soesterberg, The \\ Netherlands \\ +31346356458 \\ jan.vanerp@tno.nl
}

\author{
Peter J. Werkhoven \& \\ Marieke E. Thurlings \\ Utrecht University, Computer Science \\ Padualaan 14 \\ $3584 \mathrm{CH}$ Utrecht, The Netherlands \\ +31302534109 \\ P.J.Werkhoven@cs.uu.nl \\ M.E.Thurlings@cs.uu.nl
}

\author{
Anne-Marie M. Brouwer \\ TNO Human Factors \\ Kampweg 5 \\ 3769ZG Soesterberg, The \\ Netherlands \\ +31346356424
}

anne-marie.brouwer@tno.nl

\begin{abstract}
In this paper, we describe a Brain Computer Interface (BCI) for navigation. The system is based on detecting brain signals that are elicited by tactile stimulation on the torso indicating the desired direction.
\end{abstract}

\section{Categories and Subject Descriptors}

B.4.2 input/output devices, H.1.2 user/machine systems, I.3.6 ergonomics, J.3 life and medical sciences.

\section{General Terms}

Design, Experimentation, Human Factors, Theory.

\section{Keywords}

Perception, cognition, BMI, BCI, Neuroscience, brain-computer interface, brain-machine interface.

\section{INTRODUCTION}

As response to the threat of information overload (e.g while car driving, see Figure 1 and [1]), designers of Human Interfaces are constantly looking for new ways users and systems can interact. The search for free or underutilized communication channels has resulted in innovative displays such as virtual acoustics, vibration seats, and force feedback devices [2]. Especially when users need all their cognitive resources for the task at hand, the interface should be intuitive in a sense that it should require limited mental effort to use. An appealing example is the control device of the Nintendo Wii when used to play tennis or golf. This control device is fundamentally different from the keyboards, joysticks and gamepads to control games and that do require training or substantial mental effort.

Some developers think that the next generation of game controllers or input devices will be based on brain signals, often referred to as Brain Computer Interfaces or BCIs (see Figure 2). Navigation BCIs can be active, reactive or passive. Active BCIs require active commands of the user like the imagined movement of left hand versus right foot, or the execution of specific mental tasks like mental rotation versus tapping musical rhythms.

Copyright is held by the author/owner(s).

ICMI-MLMI'09, November 2-4, 2009, Cambridge, MA, USA. ACM 978-1-60558-772-1/09/11.
Reactive BCIs use the brain's reaction to specific stimuli and are based on the fact that this reaction differs according to aspects such as stimulus relevance and user attention. Finally, passive BCIs measure brain states such as alertness and mental workload without requiring external stimuli or specific user actions.

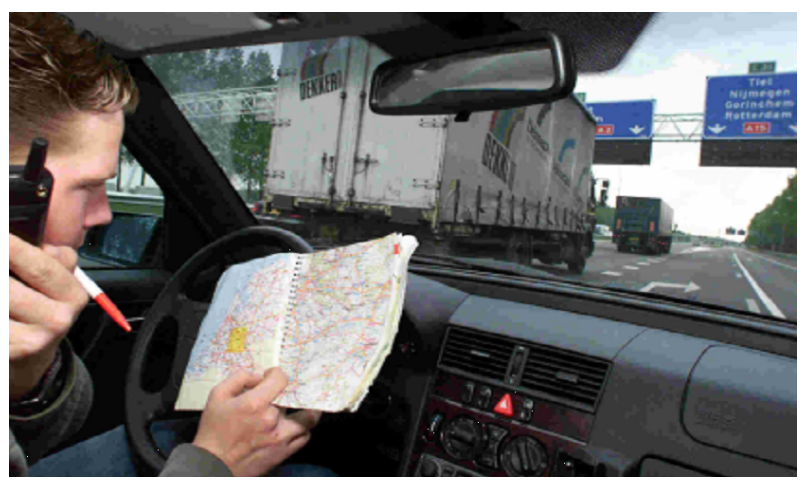

Figure 1. The risk of information overload is a major thrust for the development of innovative Human Computer Interfaces.

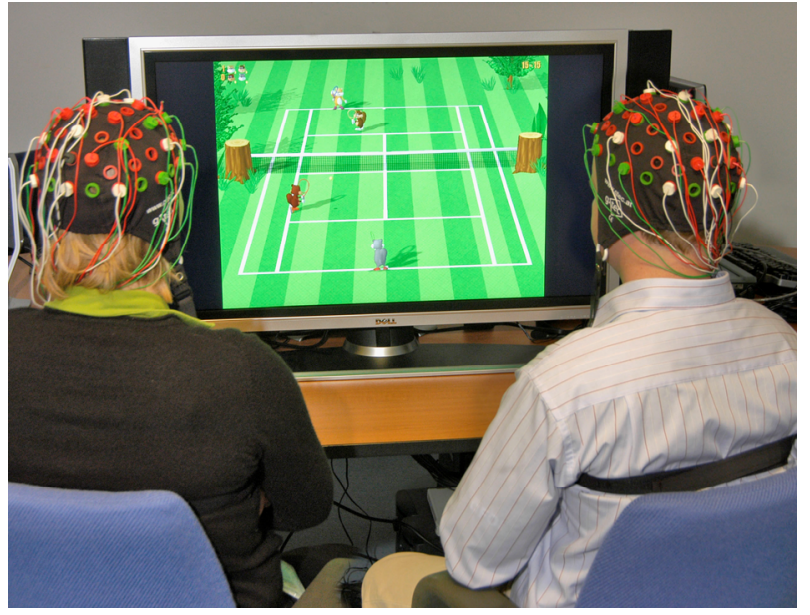

Figure 2. Gaming may be the domain that celebrates the large scale introduction of Brain Computer Interfaces for healthy users.

We believe that reactive $\mathrm{BCIs}$ are very promising to use in navigations tasks. This class of BCIs is based on reactions of the 
brain to specific stimuli and do not require specific mental commands like the common active navigation BCIs do. Therefore, reactive BCIs may have the potential to reduce the mental effort required to navigate. A second advantage is that reactive BCIs are based on brain signals that occur naturally in the brain, i.e. there is no training required. An event-related potential (ERP) is such a brain signal. An ERP is a stereotypical response to an external stimulus. A paradigm that utilizes ERPs is the oddball paradigm: rare and/or relevant events elicit a so-called P300 component of the ERP that can be detected using standard EEG equipment $[3,4,5]$.

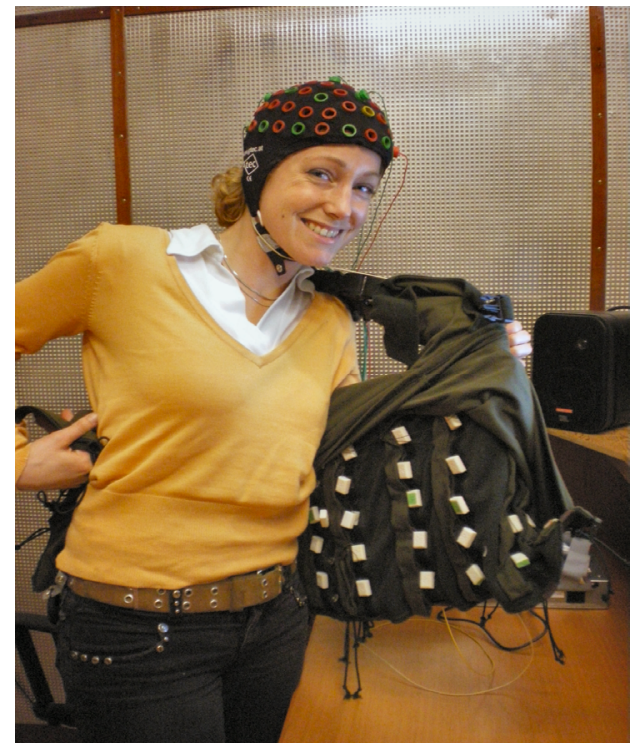

Figure 3. User showing the tactile vest that presents localized vibration around the torso to indicate different directions.

Paying attention to the vibrator indicating the desired direction will result in a brain pattern that can be detected by EEG electrodes.

\section{Reactive Brain Computer Interface}

Reactive BCIs based on the oddball paradigm generally use visual stimuli such as flashing letters or symbols. Since user attention affects the P300, the P300 ERP elicited by the target letter is easy to distinguish from those elicited by non-target letters. The disadvantage of using visual stimuli is that they add to the threat to visual overload. Therefore, we chose to present stimuli using the sense of touch, a sensory channel that is often underutilized in user-system interaction.

Figure 3 shows how our implementation of the oddball paradigm for tactile stimuli looks like. In our application, the user wears a vest or belt with vibrating tactors covering the torso. The advantages of using such a tactile torso display in a navigation display are twofold: Using the tactile channel leaves the visual channel free for other forms of communication, and tactile torso displays can present spatial information in an intuitive way. For example, a vibrotactile stimulus on the torso immediately leads to a percept of an external direction [6,7], an effect that we call the "tap on the shoulder" principle. Such an intuitive perception of external direction can also be very useful brain-based navigation interfaces $[8,9]$.

We present users with bursts of vibration delivered by one of for instance two, four, or eight tactors around the waist while he or she attends to the tactor representing the desired direction. The brain's response to the rapid stream of stimuli is measured with standard EEG equipment (g.tec medical engineering $\mathrm{GmbH}$ ) and electrodes placed at the $\mathrm{Fz}, \mathrm{Cz}, \mathrm{Pz}, \mathrm{Oz}, \mathrm{P} 3, \mathrm{P} 4, \mathrm{PO} 7$ and PO8 sites of the 10-20 system [10]. Signals go through a $0.1 \mathrm{~Hz}$ high pass-, a $60 \mathrm{~Hz}$ low pass- and a $50 \mathrm{~Hz}$ notch filter before classification. Online classification based on linear discriminant analysis is done using custom-build MatLab-based software (The Mathworks ${ }^{\mathrm{TM}}$ ).

\section{REFERENCES}

[1] Van Erp, J.B.F., and Padmos, P. 2003. Image parameters for driving with indirect viewing systems. Ergonomics, 46(15), 1471-1499.

[2] Van Erp, J.B.F. 2002. Guidelines for the use of vibro-tactile displays in human computer interaction. Proceedings of Eurohaptics 2002, pp. 18-22.

[3] Farwell, L.A., and Donchin, E. 1988. Talking off the top of your head: A mental prosthesis utilizing event-related brain potentials. Electroencephalography and Clinical Neurophysiology, 70, 510-523.

[4] Sellers, E.W., Krusienski, D.J., Mcfarland, D.J., Vaughan, T.M., and Wolpaw, J.R. 2006. A P300 event-related potential brain-computer interface (BCI): The effects of matrix size and inter stimulus interval on performance. Biological Psychology, 73, 242-252.

[5] Wolpaw, J.R., Birbaumer, N., McFarland, D.J., Pfurtscheller, G., and Vaughan, T.M. 2002. Brain-computer interfaces for communication and control. Clinical Neurophysiology 113, 767-791.

[6] Van Erp, J.B.F. 2005. Presenting Directions with a VibroTactile Torso Display. Ergonomics, 48, 302-313.

[7] Van Erp, J.B.F., and Van Veen, H.A.H.C. 2006. Touch down: the effect of artificial touch cues on orientation in microgravity. NeuroScience Letters, 404, 78-82.

[8] Brouwer, A.M. \& Van Erp, J.B.F. 2008. A tactile P300 BCI and the optimal number of tactors: Effects of target probability and discriminability. 4th International BrainComputer Interface Workshop, Graz, Austria, September 1920, 2008.

[9] Van Erp, J.B.F., van Veen, H.A.H.C., Jansen, C. and Dobbins, T. 2005. Waypoint Navigation with a Vibrotactile Waist Belt. Transactions on Applied Perception, 2 (2), 106117.

[10] Jasper, H. 1958. Report of the committee on methods of clinical examination in electroencephalography. Electroencephalography and Clinical Neurophysiology, 10, 370-375. 